\title{
The Recognition and Localization of Insulators Based on SIFT and RANSAC
}

\author{
Zhenbing Zhao, Ning Liu and Yapeng Yuan
}

\begin{abstract}
The recognition and localization of insulators is a crucial process of insulators' failure detection, especially under the complex background. With unknown insulators in test image, a method of the recognition and localization of insulators based on SIFT and RANSAC is proposed as following: SIFT (Scale Invariant Feature Transform) is used to extract a set of feature points of each insulator template image and test image. Then use RANSAC (RANdom SAmple Consensus) to remove the outliers. And each template's matched inliers are accurate points which are used to compute affine transform matrix $\mathrm{H}$ and localize the template images in test image. Thus we can detect all the template images in test image correctly. Experimental results show that the proposed approach can obtain high precision in the recognition and localization of insulators under the complex background.
\end{abstract}

Keywords: Insulators; Recognition and localization; Complex background; SIFT; RANSAC

\section{Introduction}

Insulator's [1] failure would cause huge economic losses and casualties in substation system. So it is necessary to monitor its status so as to dispose it promptly. In order to detect insulator's external defects, such as crack, surface pollution and damage, it is crucial to identify insulator from the collected scene image with complex background.

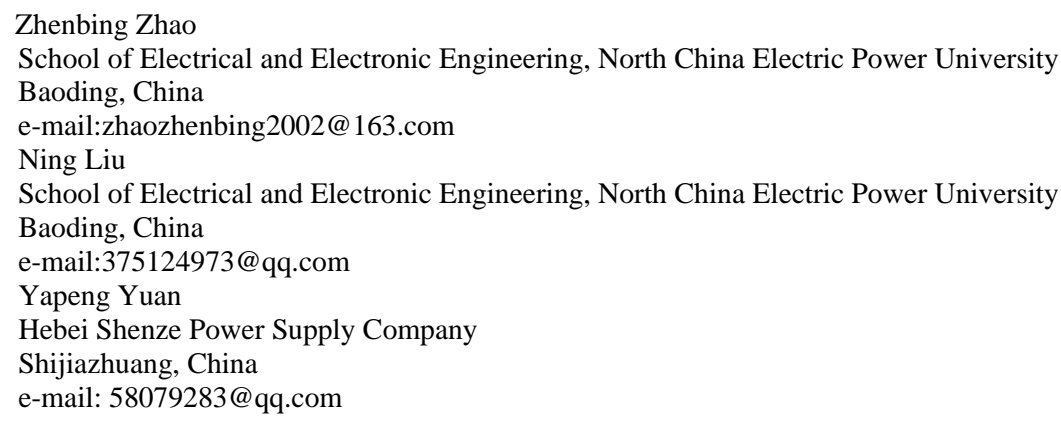


Some research has been done for recognizing insulator. Literature [2] proposes a method that integrates relative temperature distribution characteristics with Artificial Neural Network model to recognize insulators with different contamination grades. This method has a high accuracy, but it depends on experience, and has a certain degree of blindness. Literature [3] proposes a method that extracts of insulator and background based on morphological algorithm improved optimal entropy segmentation algorithm. It uses segmentation method and relies on insulator's shape feature. But it may be unable to identify damaged insulator. Literature [4] proposes a method of insulator's feature extraction based on MPEG-7 edge directions. This method can recognize insulator effectively in the complex background. But it always produces a false negative when the insulator is damaged. All these methods can recognize insulator precisely, but their application is limited, and can not solve the multiple insulators' identification under complex background with no segmentation.

This paper proposes a method of recognition and location of insulators based on SIFT and RANSAC, that can identify multiple insulators based on matching under complex background with no segmentation. Firstly, extract key feature points by SIFT, and roughly match them. Secondly, by using RANSAC attain exact matches. Finally, localize template insulators in test image by affine transformation. Then all the insulators in test image will be recognized and localized by means of iteration. This method can act as identification for the insulators' failure detection based on image processing technology.

\section{Correlation techniques}

As the technical preparation of the insulators' recognition, this chapter describes the related basic theory briefly, including SIFT algorithm, and RANSAC algorithm, and analyses the methods and problems involved.

\subsection{SIFT algorithm}

SIFT [5] is an algorithm to extract distinctive invariant features from images. The features are invariant to image scale and rotation, and even robust to noise, occlusion, shift in 3D perspective, affine transformation and change in illumination. The major steps of the SIFT algorithm are as follows:

1. Scale-space Extrema Detection

The first step is to search all scales and image locations by DoG function to identify potential key points. Points in the DoG images which are local maximum and minimum points are extracted as key points.

2. Key point Localization 
The location and scale of each candidate point is determined by a detailed model and key points with high contrast and good localized edge points are selected based on measures of stability.

3. Orientation Assignment

Orientations are assigned to each key point location based on local gradient directions. To determine the key point orientation, a gradient orientation histogram is computed in the neighborhood of the key point.

4. Key point descriptor

Sample image gradient magnitudes and orientations around each feature point and put them in an array of orientation histograms covering the region around the feature point. A 128-dimension descriptor is generated by gradients and orientations for each key point.

\subsection{RANSAC algorithm}

RANSAC [6, 7] is a robust algorithm to estimate parameters of a mathematical model from a set of observed data which contains outliers in the data set.

Specify a data set $\mathrm{P}$ composed of $\mathrm{N}$ data points, establish affine transformation model $(\mathrm{H})$ between feature points, and execute the following steps by means of iteration until the wrong feature points are eliminated completely:

1. Randomly pick up subset (S1) of four matched data points;

2. Compute the model $(\mathrm{H})$ by means of 4 selected data points;

The model between template image and test image can be represented by affine transform:

$\left(\begin{array}{l}\mathrm{x}_{2} \\ \mathrm{y}_{2}\end{array}\right)=s\left(\begin{array}{cc}\cos \theta & -\sin \theta \\ \sin \theta & \cos \theta\end{array}\right)\left(\begin{array}{l}x_{1} \\ y_{1}\end{array}\right)+\left(\begin{array}{l}t_{x} \\ t_{y}\end{array}\right)$

where $\left(x_{1}, y_{1}\right)$ and $\left(x_{2}, y_{2}\right)$ are the control point coordinates in the template image and test image, respectively, $t_{\mathrm{x}}, t_{\mathrm{y}}$ are the translation value in $x$ and $y$ direction, $\mathrm{S}$ is the scaling factor, and $\theta$ is the rotation angle.

\section{Recognition method based on SIFT and RANSAC algorithm}

\subsection{Insulators recognition method based on SIFT and RANSAC}

In order to identify multiple insulators from the complex background, a new 
method is proposed based on SIFT and RANSAC. Fig. 1 is flow chart of the method and the specific set is as following:

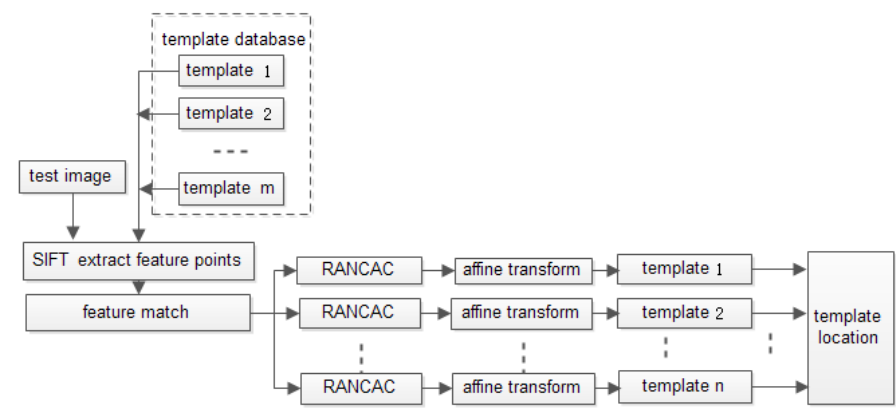

Fig. 1 The process flow diagram of the insulator's recognition

1. Image preprocess

First, the test image and template images are preprocessed with Gray Scale to convert it into gray image. Template images' lack of contrast leads to identify some useful details difficultly, so it is necessary to improve the contrast between target and background to rich image gray levels.

\section{Extract SIFT features and rough match}

Use SIFT algorithm to extract SIFT feature points of template images and test image. Then we can use the Euclidean distance between key feature vectors as similarity judgment measures in two images. And if the Euclidean distance between points is less than threshold, the points would be considered as match pair, otherwise abandon. And the points of two images are rough match.

All the template images can compose as a database and we can extract template images' features previously to match the features of test image conveniently and relatively.

\section{RANSAC discard the wrong matched points}

A complex test image may have several template images, and there may be still several incorrect matches even after rough match. RANSAC can classify a set of observed matched data which contains the wrong points, discard outliers and extract inliers to estimate parameters of a mathematical model.

\section{Affine Transformation and Localization}

The mathematical model computed by RANSAC is the transform matrix $\mathrm{H}$. Then transform template images with correct points, extract the edges of the transformed template images, and locate template insulators' edges in test image.

Execute above steps by means of iteration until all the insulators in test image have been recognized and localized. 


\subsection{Experimental results and analysis}

Take transformer bushing image as examples, and this method of the recognition and localization of insulators based on SIFT and RANSAC is proved to be effective and reliable, compared with the recognition and localization of insulators based on SIFT and LmedS [8], and with the recognition and localization of insulators based on SIFT and MLESAC [9].

\subsubsection{Identification result analysis of transformer bushing image}

Fig. 2 is the substation image, three transformer bushing images with rotation and scaling and their the binarization images with clear edges using OSTU to segment three transformer bushing images respectively, according to the big betweencluster variance between foreground and background. Use SIFT to extract key feature points and rough match. But obviously there are also some wrong match points, and use RANSAC to classify the inliers and outliers, and estimate parameters of the optimal model. And the exact match is finished. Fig. 3, 4, 5 can show the results of rough and exact match between each transformer bushing and substation image respectively. The green points are rough match points between each transformer bushing and substation image. Affine transform and all the three transformer bushing images in substation image can be localized. The localization results are shown in Fig. 6.
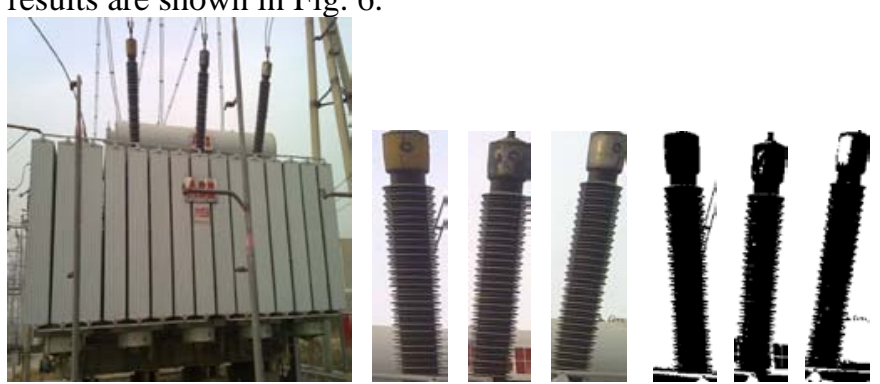

Fig. 2 Substation image, three transformer bushing images and its OSTU segmentation images

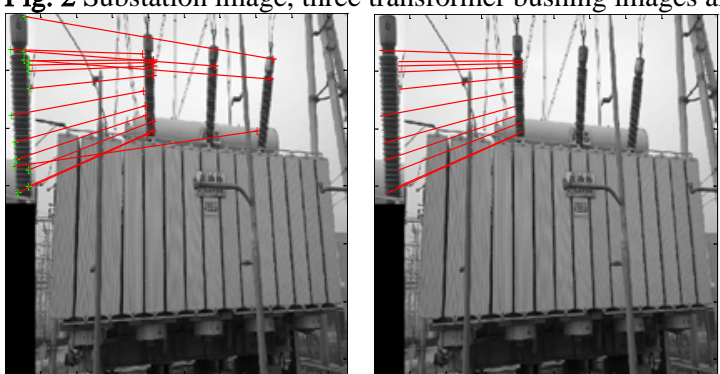

Fig. 3 The rough and exact match result of transformer bushing 1 


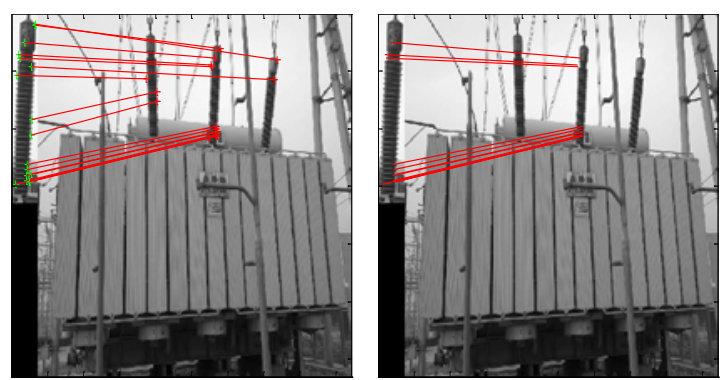

Fig. 4 The rough and exact match result of transformer bushing 2

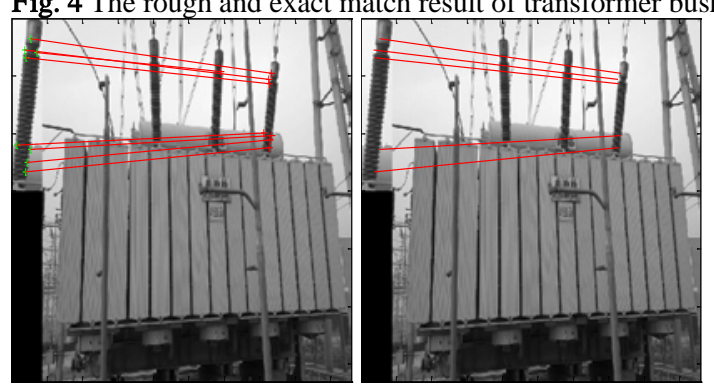

Fig. 5 The rough and exact match result of transformer bushing 3

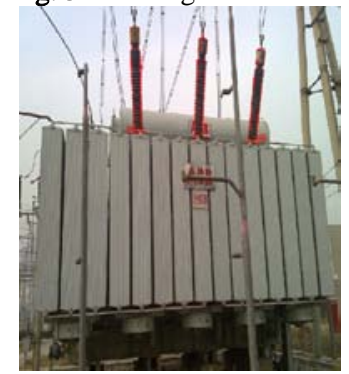

Fig. 6 Transformer bushing1, 2, 3 location results

\subsubsection{Contrast experiment}

Analyze qualitatively and compare quantitatively with two methods, the recognition and localization of insulators based on SIFT and LmedS (method 2) and recognition and localization of insulators based on SIFT and MLESAC (method 3) to confirm the superiority of this method (method 1).

(1) The results of qualitative analysis

Fig.7 is the localization of transformer bushing 3 in the substation image by method 2. The process of feature point extraction and rough match by SIFT, method 1 and method 2 are the same. Method 2 uses LmedS to remove wrong matched points. As seen in Fig.7, the matched pairs after LmedS are not the exact match pairs, and after affine transform for transformer bushing 3, localization is not exact. 

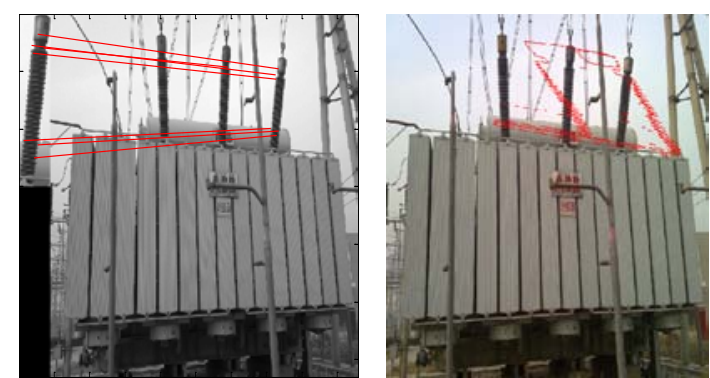

Fig. 7 Transformer bushing 3 exact match and location result by method 2

Fig. 8 is the localization of transformer bushing 3 in the substation image by method 3. Method 3 is the same as method 1 except removing wrong matches by MLESAC instead of RANSAC. Fig. 8 shows that the number of exact match points is more than method 1 compared with Fig 5, and more match radio may cause low quality of affine transform parameters, even may causes wrong localization.
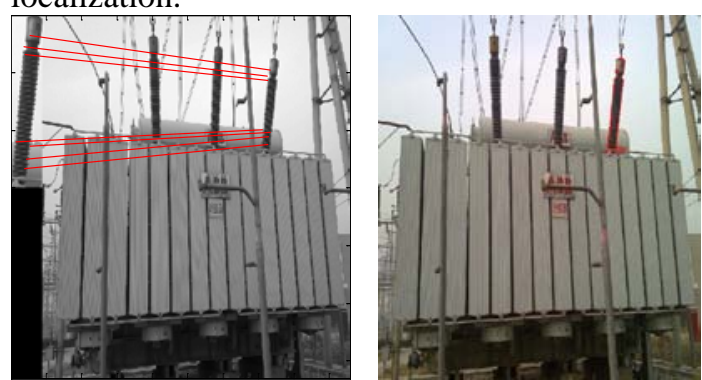

Fig. 8 Transformer bushing 3 exact match and location result by method 3

(2) The results of quantitative compare

Table 1 Transformer bushing evaluation indicators of methods

$$
\text { methods method } 1 \quad \text { method } 3
$$

\begin{tabular}{lllllcl} 
Indicators & C1 & C2 & C3 & C1 & C2 & C3 \\
\hline Repetitive rate & 0.0193 & 0.0171 & 0.0094 & 0.0193 & 0.0171 & 0.0094 \\
Match ratio & 0.647 & 0.800 & 0.625 & 0.647 & 0.800 & 0.875 \\
RMSE & 0.4262 & 0.4286 & 0.4410 & 0.1862 & 0.3485 & 1.8974 \\
\hline
\end{tabular}

$\mathrm{C} 1$, the abbreviation of transformer bushing 1; $\mathrm{C} 2$, the abbreviation of transformer bushing 2, and C3, the abbreviation of transformer bushing 3. Figures of match result show the numbers of matched points, and the indicators can be calculated according to their formula. Method 1 achieves equal repetitive rate and lower match radio, but more stable than method 3.

Form three methods of insulator recognition, method 2 can't match successfully and can't localize the insulators; the indicators show that method 1 achieves better quality than method 3 . Overall consideration, method 1 is more effective. 


\section{Conclusions}

This paper proposes a method of the recognition and localization of insulators based on SIFT and RANSAC. Firstly, use SIFT to extract the key points of template images and test image to rough match. Then remove the wrong matched points with RANSAC. Finally, by affine transform, localize template image in test image. This paper verifies the effectiveness of this method by using substation transformer bushing image. And analyze qualitatively and compare quantitatively to confirm the proposed method's superiority with two methods, the recognition and localization of insulators based on SIFT and LmedS and recognition and localization of insulators based on SIFT and MLESAC.

Acknowledgment The research is supported by "the Fundamental Research Funds for the Centra 1 Universities” under grant number 12MS122.

\section{References}

1. Jian Zhang, Ruqing Yang (2006). Insulators Recognition for 220kv 330kv High voltage Live line Cleaning robot. The 18th International Conference on Pattern Recognition(IEEE).

2. Jiangang Yao, Shilei Guan,Jiazheng Lu, et (2012). Identification of Zero Resistance Insulators by Combining Relative Temperature Distribution Characteristics With Artificial Neural Network. Power System Technology, 36(2):170-175.

3. Chunyu Yao, Lijun Jin, Shujia Yan (2012). Recognition of insulator String in Power Grid Patrol Images. Journal of System Simulation, 24(9):1818-1822.

4. Weiguo Li, Gaosheng Ye, Feng Huang,et. Recognition of insulator based on developed MPEG-7 texture feature. Image and Signal Processing, 2010,46(10):65-68

5. Lowe D G (2004). Distinctive image features from scale invariant key points. International Journal of Computer Vision, 60(2): 91-110.

6. Fisher M, Bolles R (1981). Random Sample Consensus: A Paradigm for Model Fitting with Applications to Image Analysis and Automated Cartography. Communications of the ACM, 24:381-385.

7. Wang Wei, Hong Jun, Tang Yiping (2008). Image matching for geomorphic measurement based on SIFT and RANSAC methods. In: International Conference on Computer Science and Software Engineering. Wuhan, Hubei:IEEE, 317-320.

8. Rousscuw P J, Lcroy A M (1987). Robust regression and outlier detection. New York:John Wilcy \& Sons.

9. Torr PHS, Zisserman A. MLESAC: a new robust estimator with application to estimating image geometry. Computer Vision and Image Understanding, 2000, 78(1).

10. Hong Liu, Weiguo Guo (2012). Research on Fast Parallel Image Matching Algorithm Based on Feature Points. 